\title{
Caracterização de catalisadores automotivos novos e usados visando à reciclagem dos metais
}

\section{Characterization of virgin and used automobile catalysts aiming at precious metals recycling}

Carlos Henrique Batista ${ }^{1}$; Achilles J.B. Dutra ${ }^{2}$

\author{
${ }^{1}$ Universidade Federal de Sergipe, P2CEM, São Cristóvão, SE \\ e-mail: chenriqueb@ig.com.br \\ ${ }^{2}$ Universidade Federal do Rio de Janeiro, PEMM Cidade Universitária, C.T., sala F210 - Ilha do Fundão, Rio de Janeiro, \\ RJ - Brasil - 21949-900 \\ e-mail: adutra@metalmat.ufrj.br
}

\begin{abstract}
RESUMO
A platina e os metais do grupo da platina (MGP) possuem importantes aplicações em catálise. Os catalisadores automotivos geralmente possuem platina, paládio e ródio, que são úteis na conversão dos gases nocivos produzidos durante a queima do combustível, em gases menos prejudiciais ao meio ambiente. A demanda pela platina e metais do seu grupo, aliada à sua escassez na crosta terrestre e altos valores de mercado, tornam a sua reciclagem fundamental. Neste contexto, uma vez que não existe uma formulação padronizada para a sua fabricação, a caracterização dos catalisadores automotivos é uma etapa primordial para a seleção adequada da rota de reciclagem a ser adotada. Via de regra, os catalisadores monolíticos virgens usados na indústria automotiva possuem em torno de $1,89 \mathrm{~g} / \mathrm{kg}$ de platina, $0,29 \mathrm{~g} / \mathrm{kg}$ de paládio, além de $0,10 \mathrm{~g} / \mathrm{kg}$ de ródio, metais preciosos de altíssimo valor agregado. Neste trabalho foram caracterizadas duas amostras de catalisadores automotivos, uma virgem e outra proveniente de um catalisador usado. A microscopia óptica, microscopia eletrônica de varredura com sistema de microanálise por dispersão de energia, difração e fluorescência de raios-X, além de TG-DTA foram as técnicas analíticas empregadas neste trabalho. Foi observado que os catalisadores são constituídos por uma estrutura na forma de colmeia, constituída de cordierita $\left(\mathrm{Mg}_{2} \mathrm{Al}_{4} \mathrm{Si}_{5} \mathrm{O}_{18}\right)$, recoberta com uma camada de alumina impregnada com os metais do grupo da platina. No catalisador virgem, detectou-se por fluorescência de raios-X, cerca de $0,99 \mathrm{~g} / \mathrm{kg}$ de platina, enquanto que no catalisador usado o teor de platina foi de $0,44 \mathrm{~g} / \mathrm{kg}$. Na amostra do catalisador exaurido nota-se a deposição de material carbonoso sobre a camada de alumina, além da presença de trincas, que podem levar a perdas dos metais preciosos e queda de atividade do catalisador.
\end{abstract}

Palavras-chave: platina, catalisador, reciclagem.

\section{ABSTRACT}

Platinum and platinum group metals (PGM) have important applications in catalysis. Automotive catalysts usually present platinum, palladium and rhodium. These metals play an important role in the conversion of toxic gases from fuel burn in gases less hazardous to the environment. The need for platinum and PGM allied to their scarcity in earth crust and elevated prices turn their recycling paramount. The automotive characterization is an important step for the selection of the proper recycling route, once there is no standard formulation for the manufacture of such catalysts. The virgin monolithic catalysts used by the automotive industry usually present around $1.89 \mathrm{~g} / \mathrm{kg}$ of platinum, $0.29 \mathrm{~g} / \mathrm{kg}$ of palladium besides $0.10 \mathrm{~g} / \mathrm{kg}$ of rhodium, all high valued precious metals. In this paper two automobile catalyst samples were characterized: a virgin one, provided by Umicore - Brazil and an used one, bought in the market. Optical microscopy, scanning electron microscopy (SEM) coupled with energy-dispersive X-ray spectroscopy (EDS), X-ray diffraction and fluorescence and termogravimetry coupled with differential thermal analysis (TG-DTA) were analytical techniques employed throughout this work. It was observed that the catalysts present a cordierite $(\mathrm{Mg} 2 \mathrm{Al} 4 \mathrm{Si} 5 \mathrm{O} 18)$ honeycomb structure covered with a PGM impregnated alumina layer. In the virgin catalyst around 0.99 
$\mathrm{g} / \mathrm{kg}$ of platinum was detected, while in the used one the platinum grade was $0.44 \mathrm{~g} / \mathrm{kg}$. In the used catalyst sample the presence of cracks and the deposition of carbonaceous matter onto the alumina layer were observed, which can lead to lose of activity of the catalyst.

Keywords: platinum, catalyst, recycling.

\section{INTRODUÇÃO}

A platina e os metais do grupo da platina (MGP) possuem importantes aplicações na área de catálise em função das suas propriedades físico-químicas. Entre estas aplicações, os catalisadores automotivos que geralmente possuem platina, paládio e ródio, merecem destaque. Estes metais nobres são úteis na conversão dos gases nocivos, produzidos durante a queima do combustível a formas menos tóxicas. Estes catalisadores podem ser obtidos no formato de monólitos de cordierita de magnésio $\left(\mathrm{Mg}_{2} \mathrm{Al}_{4} \mathrm{Si}_{5} \mathrm{O}_{18}\right)$, com uma estrutura em forma de colmeia. Na superfície desta estrutura é aplicado um filme de alumina- $\gamma$, impregnado de metais nobres (principalmente platina, paládio e ródio) além de outros aditivos, como o cério, para potencializar a ação catalítica e estabilizar a alumina nas condições de operação [1]].

Os gases da exaustão dos automóveis contribuem significativamente para a poluição do ar e, consequentemente, degradam o meio ambiente. Em áreas urbanas com alta concentração de veículos em circulação isso é notado pelos elevados níveis de monóxido de carbono $(\mathrm{CO})$, de dióxido de enxofre $\left(\mathrm{SO}_{2}\right)$, de compostos aromáticos e pela fumaça fotoquímica, resultante da interação entre óxidos de nitrogênio (NOx), hidrocarbonetos e luz solar, que causam irritação aos olhos, ao aparelho respiratório e danos às plantas [ㄹ-3].

Atualmente, no Brasil, os dispositivos de tratamento de gases de exaustão de veículos, por exigência da legislação, utilizam os catalisadores de três vias (TWC - Three-Way Catalyst) que podem reduzir as emissões dos gases nocivos por veículo, em mais de $90 \%$, convertendo-os em gases menos prejudiciais ao meio ambiente [4- ] ]. O uso do catalisador é obrigatório em todos os veículos automotivos, sendo regulamentado pela legislação vigente no país. A retirada, adulteração ou uso do catalisador automotivo danificado é considerado crime ambiental com sanções no Decreto Federal no 3.179 de 21 de setembro de 1999 (artigos 46 e 48) e na Lei Federal no 9.605 de 12 de fevereiro de 1998 (artigo 54), podendo variar, desde sanções administrativas à privação de liberdade [ㄱ-8].

A escolha destes metais como material catalítico resulta de três fatores: (I) os MGP tem a atividade catalítica necessária para a conversão de poluentes em curtíssimos tempos de residência, (II) são materiais catalíticos resistentes ao envenenamento por óxidos de enxofre presentes nos gases, (III) são menos propensos (mas não totalmente imunes) à inativação pela interação de altas temperaturas com os óxidos de $\mathrm{Al}, \mathrm{Ce}, \mathrm{Zr}$, etc, que constituem a superfície do filme onde estão dispersos os componentes catalíticos, também chamados de "washcoat". Inicialmente a Pt e o Pd foram utilizados como catalisadores de oxidação, mas, nos catalisadores de três vias, o Rh é também utilizado por apresentar maior atividade catalítica para a redução dos óxidos de nitrogênio [9-11].

Os catalisadores podem perder a sua atividade catalítica, como resultado de uma série de produtos químicos indesejáveis presentes originalmente nos combustíveis e por mudanças físicas [는 17$]$. Os tipos de fenômenos da inativação podem ser divididos em três categorias: química, térmica e envelhecimento mecânico [ $\underline{14}$, 15]. A inativação ocorre ao longo da vida útil do catalisador, devido a uma ou à combinação de vários fenômenos. Em qualquer caso, o efeito resultante é uma redução dos sítios cataliticamente ativos [트].

Via de regra, os catalisadores monolíticos virgens usados na indústria automotiva, possuem em torno de 1,89 $\mathrm{g} / \mathrm{kg}$ de platina, $0,29 \mathrm{~g} / \mathrm{kg}$ de paládio, além de $0,10 \mathrm{~g} / \mathrm{kg}$ de ródio; todos metais preciosos de altíssimo valor agregado. Os catalisadores usados perdem parte destes metais durante sua vida mas, ainda assim, possuem teores relevantes destes metais, muito superiores aos encontrados nos minérios [18].

A demanda pela platina e os metais do seu grupo, aliada à sua escassez na crosta terrestre e seus altos valores de mercado, tornam a reciclagem destes metais uma atividade fundamental e de grande interesse, uma vez que nos últimos anos a procura por estes metais para aplicações na indústria química e petroquímica, além do mercado automobilístico, tem crescido principalmente nos países em desenvolvimento [19].

A caracterização dos catalisadores automotivos, objeto deste trabalho, é uma etapa primordial para a seleção da rota de reciclagem a ser adotada, uma vez que não existe uma formulação padronizada para a fabricação destes. Neste trabalho, foram caracterizadas duas amostras de catalisadores automotivos: uma virgem, fornecida pela Umicore do Brasil e outra de um catalisador usado. A microscopia óptica, microscopia eletrônica de varredura com sistema de microanálise por dispersão de energia, a difração e a fluorescência de raios-X, TGDTA, além de análises químicas por espectrofotometria de absorção atômica, foram as técnicas analíticas 
usadas neste trabalho.

\section{MATERIAIS E MÉTODOS}

\subsection{Microscopia óptica}

As imagens para estudo da morfologia foram obtidas por microscopia eletrônica de varredura com elétrons secundários e a detecção dos metais e contaminantes na estrutura da cordierita foi realizada por microanálise no equipamento Jeol JSM 6460LV, equipado com detectores de energia dispersiva de raios X (EDS). As amostras do catalisador automotivo foram embutidas em resina, lixadas, polidas, e recobertas por carbono.

\subsection{Microscopia eletrônica de varredura (MEV) com microanálise}

A análise morfológica foi realizada a partir da obtenção de imagens, com aumento de $50 \mathrm{X}$, através de um microscópio óptico Pro-scope HR, acoplado a microcomputador.

\subsection{Difração de raios $X$}

Os difratogramas de raios $\mathrm{X}$ das amostras, na forma de pó, foram obtidos em um difratômetro Rigaku, operando em modo de varredura, com radiação de $\mathrm{Cu}-\mathrm{K} \alpha\left(\lambda=1,5418 \AA \AA^{\circ}\right)$ e filtro de níquel com voltagem de 40 $\mathrm{KV}$ e corrente de $40 \mathrm{~mA}$. A velocidade de varredura utilizada foi de $5^{\circ} / \mathrm{min}$ em $2 \theta\left(5\right.$ a $\left.80^{\circ}\right)$.

\subsection{Fluorescência de raios $X$}

A composição química dos catalisadores automotivos foi determinada por meio de análises realizadas com fluorescência de raios X em um equipamento SRS303 Bruker, operado a uma potência fixa de $40 \mathrm{kV}$ e 30 $\mathrm{mA}$.

\subsection{Análise térmica}

A análise termogravimétrica foi realizada em um TA Instruments 2960. SDT V3.0F, que operou com fluxo de $\operatorname{ar}(100 \mathrm{~mL} / \mathrm{min})$ e com uma taxa de aquecimento de $10^{\circ} \mathrm{C} / \mathrm{min}$.

\section{RESULTADOS E DISCUSSÃO}

Esta seção deverá apresentar todos os resultados obtidos com as informações necessárias para se verificar com segurança os valores medidos e correspondente significância. Muito embora os resultados possam ser apresentados juntos com a discussão do seu significado, é sugerido que uma profunda discussão, baseada em informações da literatura, seja apresentada em uma seção separada.

Cuidar para que na apresentação de todos os resultados experimentais tenha uma análise estatística, incluindo desvio padrão das medidas e barras de erro nos gráficos.

Um ou mais anexos podem ser usados para mostrar detalhes dos resultados analisados que venham a tornar a seção sobrecarregada.

\subsection{Microscopia óptica}

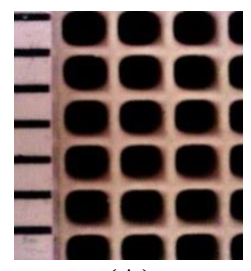

(A)

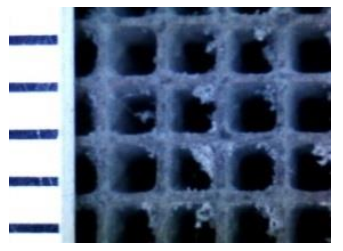

(B)

Figura 1: Aspectos típicos, obtidos por microscopia óptica, de catalisadores automotivos virgem (A) e usado (B). Escala lateral em $\mathrm{mm}$. 
O aspecto típico, obtido por microscopia óptica, de catalisadores automotivos virgens (A) e usado (B) é apresentado na Figura 1. Pode ser observada uma estrutura tipo colmeia, com alvéolos de seção quadrada com aresta de aproximadamente $1 \mathrm{~mm}$. No catalisador usado, pode ser observada ainda a presença de depósitos decorrentes de produtos de combustão, que podem afetar a eficiência do catalisador.

\subsection{Microscopia eletrônica de varredura (MEV) com microanálise}

Mev $230909(3)$

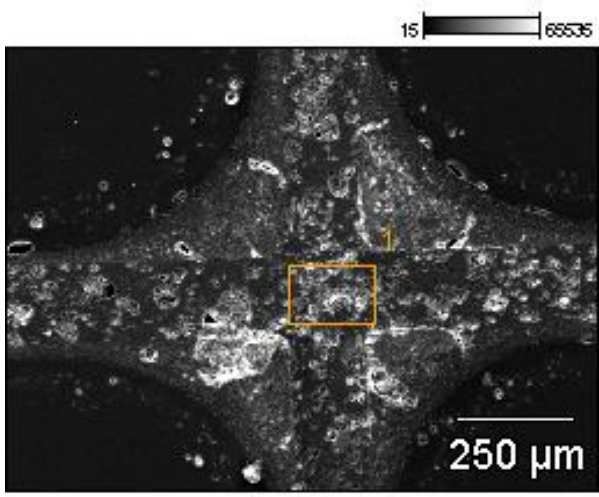

(A)

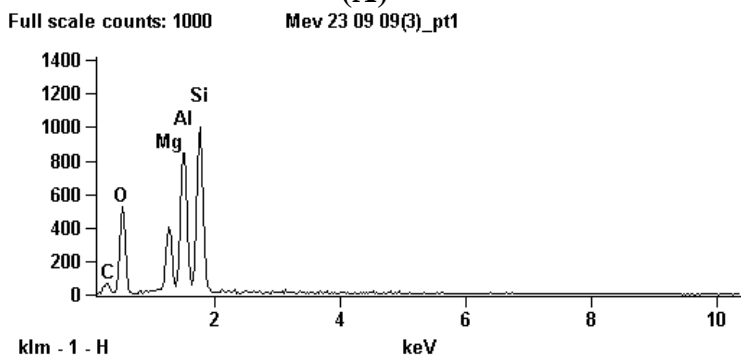

Mev 230909

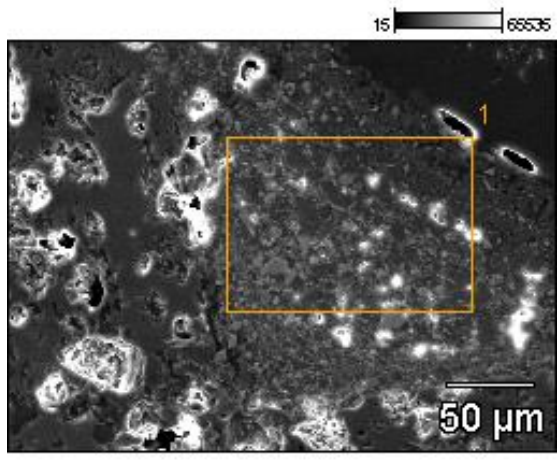

(C)

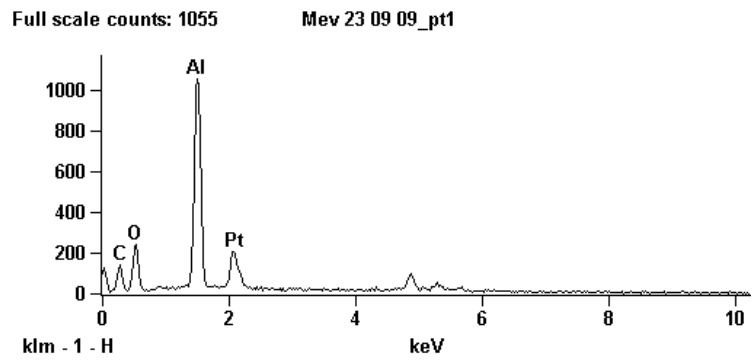

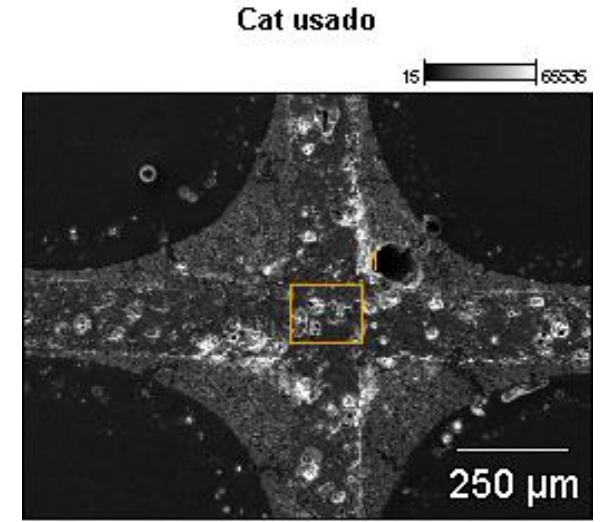

(B)

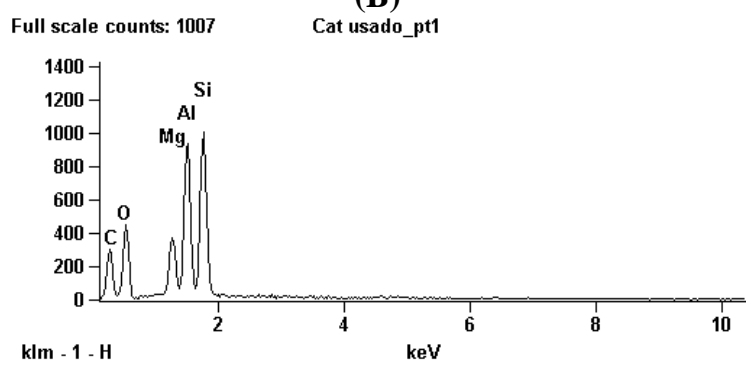

Cat usado(1)

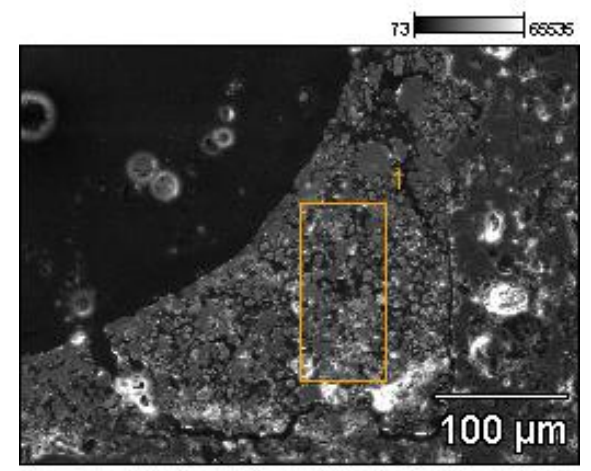

(D)

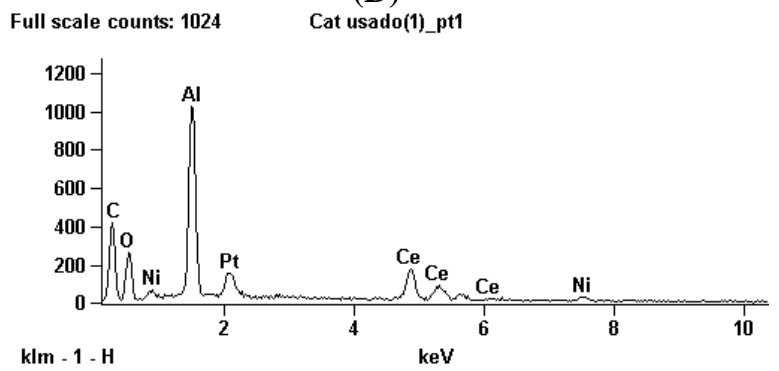

Figura 2: Aspecto típico, obtido por MEV, da matriz de catalisador novo (A) e usado (B) com respectivos EDS e detalhe do revestimento (washcoat) de catalisador novo (C) e usado (D) com respectivos EDS.

O aspecto típico, obtido por MEV da matriz de catalisador novo (A) e usado (B) com respectiva EDS (micro- 
análise por dispersão de energia) e detalhe do revestimento (washcoat) de catalisador novo (C) e usado (D) com respectiva EDS é apresentado na Figura 2. Pode ser observado que a matriz dos catalisadores novos e usados não apresentam alterações significativas e que ela é constituída de oxigênio, magnésio, alumínio e silício, elementos constituintes da cordierita. Nos catalisadores usados, o pico de carbono é mais intenso, indicando a deposição de produtos de combustão que podem levar à perda de atividade catalítica, conforme relatado por Rumpold e Antrekowitsch [20]. Pode ser observado ainda que o washcoat do catalisador usado apresenta-se com trincas e sinais de deterioração, indicando que este revestimento pode ser perdido parcialmente durante o uso do catalisador, fato já comprovado por meio de análises de poeiras encontradas em estradas e avenidas com tráfego intenso, que constataram a presença de metais do grupo da platina, normalmente presentes neste material [21] , como mostra o EDS das Figuras 2(C) e 2(D).

Os espectros das Figuras 2(C) e 2(D) indicam que o washcoat usado nas amostras de catalisador estudadas são constituídos de óxido de cério e de alumínio, nos quais está presente a platina, e eventualmente os metais de seu grupo, que aqui não estão em concentração suficientemente elevada para serem detectados por esta técnica. Contudo, as micrografias indicam que o aspecto estrutural dos catalisadores demanda uma moagem fina para viabilizar a liberação dos metais do grupo da platina.

\subsection{Microscopia eletrônica de varredura (MEV) com microanálise}

A comparação de difratogramas de raios $\mathrm{X}$ obtidos com amostras de catalisadores usados e virgens com padrão de cordierita é apresentada na Figura 3. Pode ser observado que, tanto no catalisador virgem como no usado, os picos mais intensos estão associados à matriz de cordierita e que todos os metais nobres, isto é, Pt, $\mathrm{Pd}$ e $\mathrm{Rh}$, se apresentam tanto na forma de óxidos como na forma metálica.

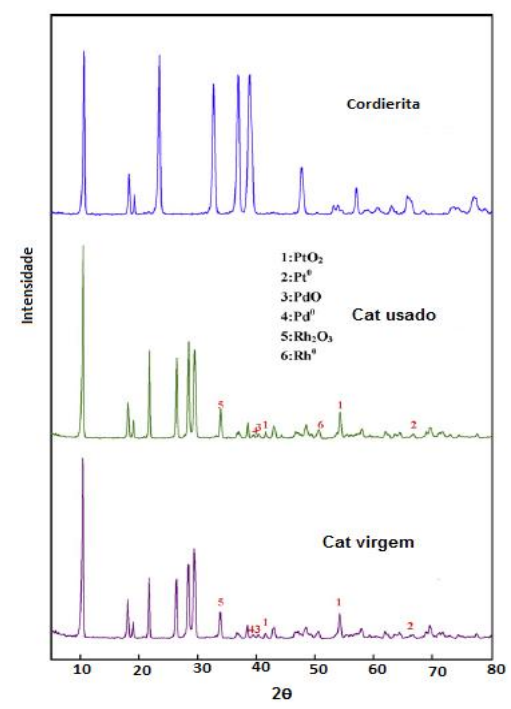

Figura 3: Comparação de difratogramas de raios-X obtidos com amostras de catalisadores usado e virgem com padrão de cordierita.

\subsection{Microscopia eletrônica de varredura (MEV) com microanálise}

Análises de fluorescência de raios $\mathrm{X}$ de catalisadores novos e usados são apresentadas respectivamente na Tabela 1. Pode ser observado que no catalisador novo não foi detectada a presença de paládio. No catalisador novo detectou-se uma quantidade de platina superior ao do catalisador usado, fato que pode ser atribuído tanto à procedência dos catalisadores como também à perda do washcoat pelo uso do catalisador, devido ao arraste pelos gases de combustão [22]. A presença de ródio não foi detectada por esta técnica em nenhuma das amostras, possivelmente por estar abaixo do limite de detecção. 
Tabela 1: Análise do catalisador novo e usado $(\% \mathrm{~m} / \mathrm{m})$

\begin{tabular}{c|c|c}
\hline & Catalisador novo & Catalisador usado \\
\hline $\mathrm{Al}_{2} \mathrm{O}_{3}$ & 53,872 & 46,234 \\
$\mathrm{SiO}_{2}$ & 19,317 & 30,029 \\
$\mathrm{ZrO} 2$ & 8,471 & 3,136 \\
$\mathrm{CeO}_{2}$ & 7,227 & 7,205 \\
$\mathrm{MgO}$ & 4,655 & 7,514 \\
$\mathrm{P}_{2} \mathrm{O}_{5}$ & 3,813 & 0,754 \\
$\mathrm{NiO}$ & 0,598 & 1,547 \\
$\mathrm{La}_{2} \mathrm{O}_{3}$ & 0,529 & 0,606 \\
$\mathrm{Fe}_{2} \mathrm{O}_{3}$ & 0,425 & 1,148 \\
$\mathrm{TiO}_{2}$ & 0,275 & 0,386 \\
$\mathrm{PtO}_{2}$ & 0,115 & 0,051 \\
$\mathrm{SO}_{3}$ & - & 0,473 \\
$\mathrm{CaO}$ & - & 0,329 \\
$\mathrm{PdO}$ & - & 0,278 \\
$\mathrm{~K}_{2} \mathrm{O}$ & - & 0,180 \\
$\mathrm{ZnO}$ & - & 0,103 \\
$\mathrm{PbO}$ & - & 0,027 \\
$\mathrm{SrO}$ & - & 0,000 \\
\hline
\end{tabular}

\subsection{Microscopia eletrônica de varredura (MEV) com microanálise}

A análise termogravimétrica e térmica diferencial de uma amostra de catalisador automotivo usada é apresentada na Figura 4. Podem ser observadas duas regiões de perda de massa em função da temperatura. A primeira, para temperaturas inferiores a $150^{\circ} \mathrm{C}$, deve estar associada à perda de umidade da amostra, que é caracterizada por uma reação endotérmica, causando um ligeiro decréscimo na temperatura; a segunda, mais expressiva, para temperaturas na faixa de 300 a $900^{\circ} \mathrm{C}$, deve estar associada à perda de matéria volátil do material carbonoso, depositado sobre o catalisador, em decorrência da combustão e também à oxidação deste material para as temperaturas mais elevadas, isto é, acima de $550^{\circ} \mathrm{C}$, caracterizada por uma reação exotérmica.Estes resultados indicam que a calcinação deste material pode vir a ser um tratamento importante para remover material carbonoso da superfície dos metais preciosos. Esta operação pode vir a ser importante para expor os metais do grupo da platina a um agente lixiviante, no caso de um possível processamento hidrometalúrgico, como observado anteriormente por outros pesquisadores [23]. 


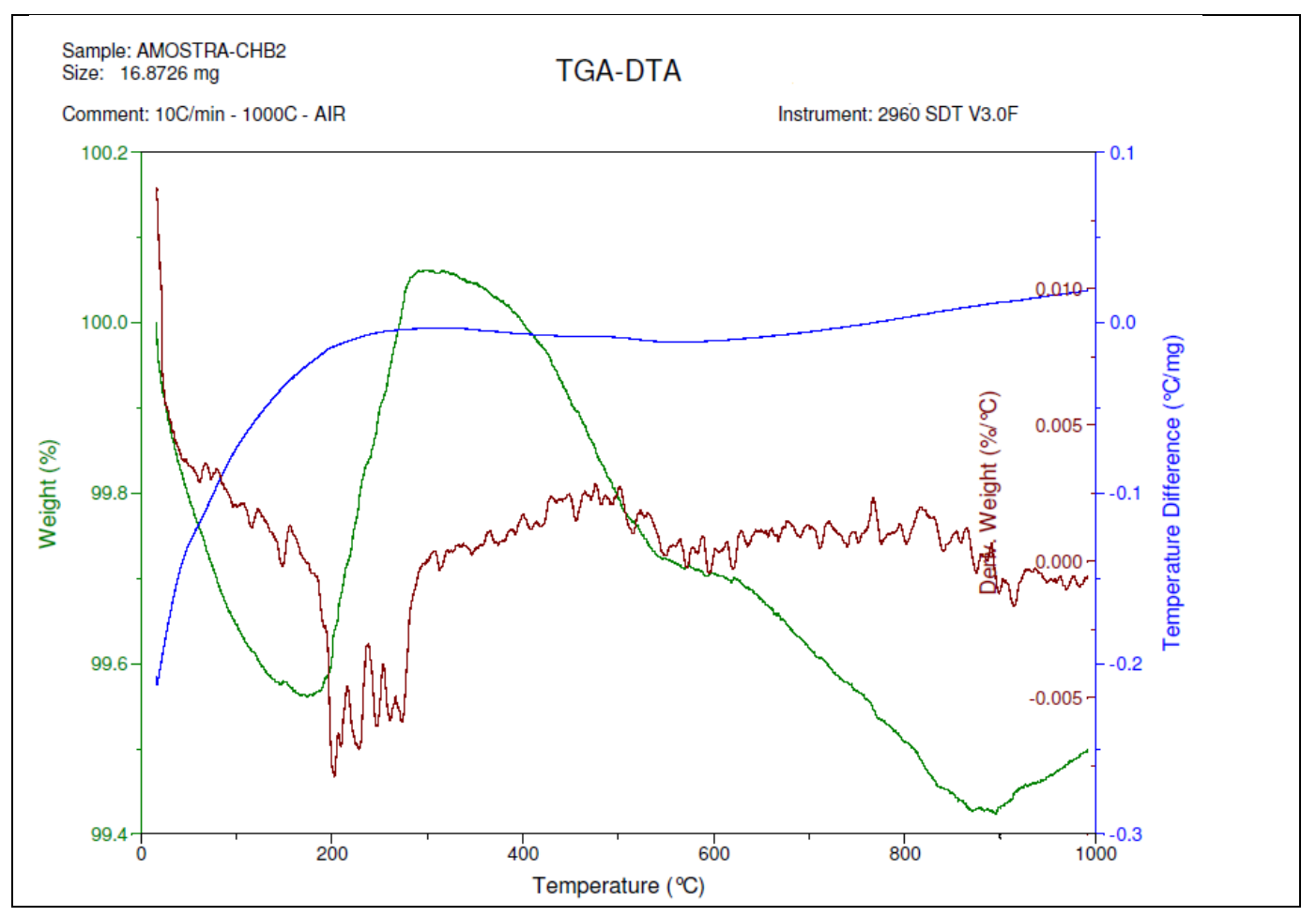

Figura 4: Análise térmica diferencial e termogravimétrica de amostra de catalisador automotivo usado.

\section{CONCLUSÕES}

Foi observado que os catalisadores usados, via de regra, possuem menor teor de metais preciosos que os catalisadores novos, porém ainda assim o seu teor é relevante para a reciclagem.

Foi observado que os catalisadores usados podem perder sua eficiência devido à redução do teor de metais preciosos e também pela deposição de material carbonoso sobre eles.

Testes de análise térmica indicaram que a calcinação prévia do material pode vir a ser um pré-tratamento importante para a remoção de material carbonoso depositado no catalisador.

\section{AGRADECIMENTOS}

Os autores agradecem ao CNPq, CAPES e FAPERJ pelo apoio financeiro.

\section{BIBLIOGRAFIA}

[1] SHYU, J. Z., OTTO, K., WATKINS, W. L. H., et al., "Characterization of Pd/ $\gamma$-alumina catalysts containing ceria", J. Catal., v. 114, n.1, pp. 23-33, 1988.

[2] LEACH, B. E., Applied Industrial Catalysis, Orlando, Academic Press, 1984.

[3] ACRES, G. J. K. "Perspective in Catalysis", Oxford, Blackwell Scientific Publications, 1992.

[4] ISMAGILOV, Z. R., SHKRABINA, R.A., KORYABKINA, N. A., et al., "Preparation and study of thermally stable washcoat aluminas for automotive catalysts", Studies in Surface Science and Catalysis, v. 116, pp. 507-511, 1998.

[5] MORTERRA, C., MAGNACCA, G., BOLIS, V., et al., "Structural, morphological and surface chemical features of Al2O3 catalyst supports stabilized with CeO2", Studies in Surface Science and Catalysis, v. 96, pp. 361-373, 1995.

[6] SHYU, J. Z., OTTO, K., "Identification of platinum phases on $\gamma$-alumina by XPS", Applied Surface Science, v. 32, n. 1-2, pp. 246-252, 1988.

[7] BRASIL. Lei $n^{\circ} 9.605$, de 12 de fevereiro de 1998. Dispõe sobre as sanções penais e administrativas derivadas de condutas e atividades lesivas ao meio ambiente. Legislação Federal. www.planalto.gov.br. Acessado em fevereiro de 2010.

[8] BRASIL. Lei no 8.723, de 28 de outubro de 1993. Dispõe sobre redução da emissão de poluentes por 
veículos automotores. Legislação Federal. www.planalto.gov.br. Acessado em fevereiro de 2010.

[9] MEGUERIAN, C., HIRSCHBERG, E., RAKOVSKY, F., Catalyst for treating exhaust gas from internal combustion engine, US Patent 4006103, Feb. 1, 1977.

[10] TAYLOR, K.C., “Automobile Catalytic Converters", Studies in Surface Science and Catalysis, v. 30, pp. 97-116, 1987.

[11] MURAKI, H., SOBUKAWA, H., KIMURA M.,et al., Society of Automotive Engineers Conference, Paper No. 900610, 1990.

[12] LUI, Y. K.., DETTLING, J. C., Society of Automotive Engineers Conference, Paper No. 930249, 1993.

[13] BUTT, J.B., PETERSEN, E. E., Activation, deactivation and poisoning of catalysts. New York, Academic Press, 1988.

[14] FORZATTI, P., LIETTI, L., "Catalyst deactivation”, Catalysis Today, v. 52, n. 2-3, pp.165-181, 1999.

[15] BARTHOLOMEW C.H., "Catalyst deactivation and regeneration", In: Kirk-Othmer Encyclopedia of Chemical Technology, 5 ed., John Wiley \& Sons, v. 5, pp. 255-322, 2004.

[16] BARTHOLOMEW C.H., "Mechanisms of catalyst deactivation", Applied Catalysis A: General, v. 212, n.1-2, pp. 17-60, 2001.

[17] MOULIJN J.A., VAN DIEPEN A.E., KAPTEIJN F., "Catalyst deactivation: Is it predictable? What to do?", Applied Catalysis A: General, v. 212, n. 1-2, pp. 3-16, 2001.

[18] SHELEF, M., MCCABE, R. W., "Twenty-five years after introduction of automotive catalysts: what next?", Catalysis Today, v. 62, n. 1, pp. 35-50, 2000.

[19] Platinum 2010, John Mathews, pp. 23-30, 2010.

[20] RUMPOLD, R., ANTREKOWITSCH, J., "Recycling of platinum group metals from automotive catalyst by an acidic leaching process", In: Proceedings of the 5th International Platinum Conference, Sun City, South Africa, The Southern African Institute of Mining and Metallurgy, pp. 695-713, 2012.

[21] SCHAFER, J., PUCHELT, H., "Platinum-Group-Metals (PGM) emitted from automobile catalytic converters and their distribution in roadside soils". Journal of Geochemical Exploration, v. 64, n. 1-3, pp. 307314, 1998.

[22] PRICHARD, H.M., SAMPSON, J., JACKSON, M., "A further discussion of the factors controlling the distribution of Pt, Pd, Rh and $\mathrm{Au}$ in road dust, gullies, road sweeper and gully flusher sediment in the city of Sheffield, UK”, Science of the Total Environment, v. 407, n.5, pp. 1715-1725, 2009.

[23] ABERASTURI, D. Jimenez de., PINEDO, R., LARRAMENDI, I. Ruiz de., et al., "Recovery by hydrometallurgical extraction of the platinum-group metals from car catalytic converters", Minerals Engineering, v. 24, n.6, pp. 505-513, 2011. 